\title{
Population density and nonaggressive competition
}

\author{
MICHAEL LOBB \\ Graduate School of Social Work, The University of Texas at Arlington, Arlington, Texas 76019
}

and

GARVIN McCAIN

Department of Psychology, The University of Texas at Arlington, Arlington, Texas 76019

\begin{abstract}
The Calhoun (1962) paper has become a keystone article for the population density literature. Although a few research reports have followed, several aspects of population density and the behavioral phenomena identified by Calhoun have been ignored. For example, the consum. matory behavior in-aggregate property of the behavioral sink is an unstudied phenomenon. Furthermore, although the literature on potential population reduction mechanisms has grown rapidly, the mechanisms of pup mortality (cannibalization by adults) as identified by Calhoun has not been pursued systematically. Finally, the question of whether social or spatial density or other factors constitute the potent variables in the development of social pathology has not been tested directly. The purpose of these five related experiments was to investigate aspects of these behaviors.
\end{abstract}

The effects of crowding in both nonhumans (e.g., Esser, 1971) and human beings (e.g., Wohlwill \& Carson, 1972) have become popular research topics since the classic work of Calhoun (1962). Interestingly enough, although Calhoun's work has had great influence, there has been relatively little work on some of the specific problems posed by his studies. One important concept growing out of his work was that of a behavioral sink. In general, the behaviors associated with a behavioral sink differ from those usually encountered. Some of these differences were seen in sexual behavior, social organization, aggression, maternal behavior, and feeding. All the studies in this paper deal with some problem raised by Calhoun's work or are extensions thereof. Some aspect of this behavioral sink concept is examined in each study.

In Experiment 1, crowding is broken down into two components, spatial density (animals per square meter) and social density (possible number of social interactions). The effects were measured in terms of aggression, natality, and mortality. Additional observations were made on the appearance of drinking competition.

In Experiment 2, females removed from the crowded conditions were placed in single cages and bred. The object was to examine the possibility that the prior high pup mortality rate was due to a lack of parental care. Further, the study allowed examina-

Portions of this paper were presented at the Psychonomic Society convention in November 1976. tion of the problem of whether maternal deficits in the crowded condition were temporary or long term.

Both social and spatial density were decreased in Experiment 3. In addition, the possibility of malemale aggression was eliminated. Natality and mortality of pups was examined in this context.

Experiment 4 also centered around pup natality and mortality. Three degrees of nest protection were employed. Pups born outside the group cage were added at different ages to examine factors related to the cessation of cannibalization.

Competition in eating and drinking was the focus of Experiment 5. Movable and fixed sources of food and water were utilized. ${ }^{1}$

\section{EXPERIMENT 1: SOCIAL AND SPATIAL DENSITY, AND SOCIAL ORGANIZATION}

Several authors (Altman, 1975; Cox, Paulus, McCain, \& Schkade, in press; Paulus, Cox, McCain, \& Chandler, 1975; Stokols, 1972; Fidell, Lopoz, \& Waddell, Note 1) have distinguished between social and spatial density as effective potential variables in the social pathology of populations. The distinction is made that spatial density is a physical variable expressed by the number of organisms per unit of area, while social density is a psychological variable that at a minimum must include an expression of the number of possible social pairings and/or interactions in a specified situation. Ten animals in $10 \mathrm{sq} \mathrm{m}$ would have the same spatial density as 100 animals in 
$100 \mathrm{sq} \mathrm{m}$ although the social density would be exponentially different between the two conditions. Fidell et al. (Note 1) investigated social and spatial density in rat populations with free access to an ethanol solution. The amount of alcohol consumed was taken as a measure of stress. This measure indicated that both high spatial density and high social density promoted stress. Although this result points to the importance of the social density variable, it fails to separate spatial from social density effects. The purpose of Experiment 1 was to increase social density while holding spatial density constant and to employ an array of behavioral measures in an attempt to assess the role of the social density variable.

\section{Method}

Subjects. The subjects were 27 male and 27 female Long-Evans rats varying in age from 100 to 250 days. Twenty-seven animals (13 male, 14 female) were purchased from Simonsen Laboratories; the other 27 (14 male, 13 female) were reared in the psychology laboratories at the University of Texas at Arlington. Laboratoryreared animals were maintained in single cages after weaning at age 21 days. Litter reduction or the selection of pups was not employed; instead, the 27 animals were randomly selected from among the weaned adults. During the course of this experiment, 8 adult animals died and were not replaced.

Apparatus. Housing consisted of wood and wire-mesh cages with a common depth of $45 \mathrm{~cm}$ and $1 \mathrm{sq} \mathrm{m}$ of surface area per cage (.037 sq $\mathrm{m}$ per animal). These adjacent cages were built on $1-m$ legs and had wire-mesh tops and bottoms with wooden sides. Along one side of each cage, seven 1-liter water bottles were arranged with $13 \mathrm{~cm}$ of separation between nozzles. Ad-lib diet of Purina Lab Chow was provided, the food pellets being dispensed once a day on the floor of the cages along with an abundant supply of torn strips of paper towels. A removable wood partition separated the two cages. The mean ambient temperature was $72^{\circ} \pm 2^{\circ} \mathrm{F}\left(22^{\circ} \pm 1^{\circ} \mathrm{C}\right)$ and the humidity was measured at $29 \% \pm 4 \%$. Constant ( $24 \mathrm{~h} /$ day) overhead lighting was provided from fluorescent bulbs.

Procedure. Since five experiments were conducted in this series, a brief summary of the procedures is included in Figure 1 . The procedures in Experiment 1 are divided into three phases:Phase 1, October 23-November 25; Phase 2, November 26-January 7, which differed with respect to social density conditions; followed by a 14-week Phase 3, January 8-June 3, which was a continuation of the second phase. During Phase 1, the animals were housed in the two physically isolated, but adjacent, cages with one, 27 iubject, sexually heterogeneous group in each cage. Thus, each zroup had access to the auditory and olfactory cues of the other zroup. In order to include visual cues in this stimulus array, he partition separating the cages was removed and replaced with I wire screen during the last week of Phase 1 . Phase 2 was initiated y removing the wire screen so that the 53 subjects $(1$ female ubject died during Phase 1 of Experiment 1) had access to the ntire area. The purpose of this manipulation was to maintain patial density constant at 26.5 animals/sq $\mathrm{m}$ while increasing he number of possible social pairings. According to the formula $\mathrm{N}(\mathrm{N}-1) / 2$ ] provided by Carpenter (1940), the number of otential intragroup relations increased from 351 to 1,431 while patial density was held constant. At the beginning of Phase 3 January 8 ), 18 of the females and 1 of the males were removed nd replaced by 18 naive females. The removed females were used 1 Experiment 2 and the male was used in Experiment 3.

The frequency of aggressive behaviors was measured during the Jllowing periods; Phase 1, November 19-25; Phase 2, November 6-29, December 2-3; and twice during the final week of Phase 3,
May 27-29, May 30-June 1. Comparisons between the measures in Phase 1 and Phase 2 were designed to measure the incidence of aggression as a function of the degree of social density under conditions of constant spatial density. For the purpose of measuring aggression in the last week of Phase 3, the remaining 46 subjects (8 died; see results section) were separated into two sexually heterogeneous groups of 23 subjects divided by a wire screen on May 23. Selection of individuals for the formation of the two groups was random in the case of the females, and in the case of males it was based on position in the dominance hierarchy. Males were ranked in dominance according to frequency of aggression. Matched halves of the male population based on dominance were included in each group. The groups were maintained in the separate condition for 6 days and then recombined for 1 week.

The observations were conducted by two individuals. In order to establish observer reliability, the periods of preexperimental observation were performed jointly until agreement between observers was consistent. Consistency between individuals when observing small numbers of pilot animals was $100 \%$. The consistency between observers when taking simultaneous measurements from large numbers of animals (27 to 54) rarely exceeded $70 \%$. The volume of behavior from large groups simply exceeds the ability of individuals to record. All observations subsequent to the preexperimental period were carried out singly with the use of individual observers balanced across experimental conditions. Observers were trained to identify periods of sexual activity associated with estrus and postpartum estrus. The object was to distinguish sexual activity from nonsexual aggression.

Although the observation periods were 20,30 , or $40 \mathrm{~min}$ in length, the frequencies of agonistic behaviors in $10 \mathrm{~min}$ were calculated and used as the basic data unit.

Two types of observations were conducted during both phases: frequency of aggressive behaviors and vital statistics. Observations on aggressive behaviors consisted of identifying and recording the incidence of eight aggressive motor patterns: rearing, rearing with pawing, rearing with vocalization, attempted or actual biting, back lying (appeasement), escape jumping, and presenting the flanks. These behaviors were identified and selected for observation based in part on descriptions by Barnett and Evans (1965) and EiblEibesfeldt (1961) and in part on preexperimental records. Rearing was scored if two animals rose on the hind paws and faced each other. If such an action was accompanied by repeated contact with the front paws, a rearing-with-pawing was scored but rearing alone was not scored. Similarly, if vocalization with rearing was observed, rearing with vocalization was scored and no other count of rearing was taken. Biting was scored if, during the occurrence of some agonistic motor pattern, an animal, with its mouth open,

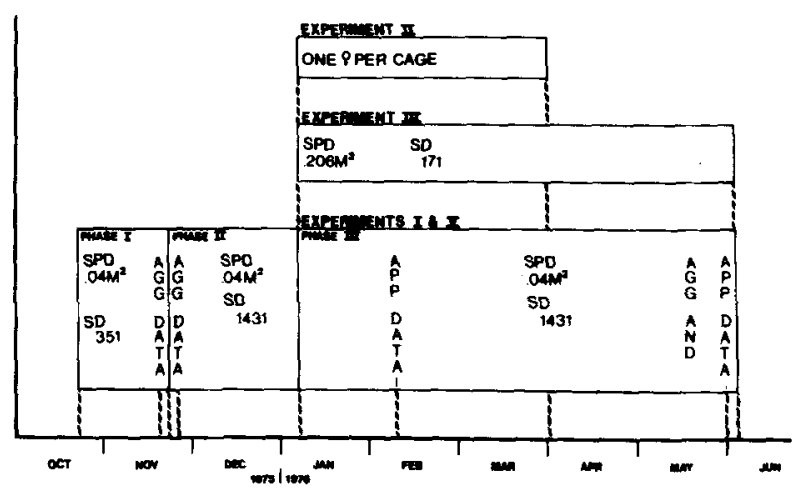

Figure 1. Phases with times of collection aggression (AGG) data, appetitive (APP) data, and social (SD) and spacial (SPD) density conditions (Experiments 1, 2, 3, and 5). 
made contact with or attempted contact with another animal. Biting during sexual behaviors such as mounting was not scored as aggressive behavior. Back lying was scored if during the interchange of other agonistic behaviors one animal lay on its back, extended its limbs upward, and remained relatively rigid. Escape jumping consisted of leaping during the course of executing other agonistic motor patterns and included running while being pursued after jumping. Presenting the flanks consisted of a lateral approach with the back raised or arched. Such movements were most often followed by withdrawal on the part of the animal being approached. A record of the frequency of occurrence was kept on each agonistic behavior. The incidence and location of wounds or fresh scars were also recorded.

In addition to observations on wounds and agonistic behaviors, records were kept on vital statistics, including mortality and natality. Three types of mortality were identified and recorded. The cannibalization of pups was recorded when an incident was directly observed or if partial remains were found. Adult mortality was categorized as either an incidence of an intact carcass or an injured carcass with the area and type of injury noted. Natality measures included the observed incidence of litters and the number of pups, although these were only approximate due to the high rate of cannibalization.

\section{Results}

Aggression. In Phase 1, the incidence of presenting the flanks was .76/10-min observation period, while in the Phase 2 combining of groups, this rate rose to 2.36/10-min observation period. Compared on the basis of a Mann-Whitney $U$ test, the phases were significantly different $[\mathrm{U}(7,7)=8.5, \mathrm{p}<.025]$. The incidence of aggression summed across the eight motor patterns increased significantly from Phase 1 to Phase $2[\mathrm{U}(7,7)=10, \mathrm{p}=.036]$.

Measures of aggression during separation and recombination in Phase 3 (May 27-June 1) were significantly different only at the $20 \%$ level.

Natality. Observed natality was also affected by the experimental manipulation. During the 3 weeks immediately preceding Phase 2 , seven litters were observed and the period of time between litters varied from 1 to 7 days. Following the experimental manipulation (combination), no litters were observed for 19 days. Thereafter, the rate of observed litters approached the preexperimental rate with 10 litters occurring within 3 weeks and at least 1 litter every 7 days.

Mortality. Adult mortality during Phase 1 consisted of one female whose carcass showed no signs of cannibalization, while three adults were cannibalized during the first week of Phase 2. Furthermore, 12 of the 29 observed pups in Phase 1 lived beyond $24 \mathrm{~h}$, while none of the 15 pups observed in Phase 2 survived for $24 \mathrm{~h}$. In two data collection periods, pups were observed to be cannibalized as they were delivered by the mother. Finally, during Phase 3, no evidence of pup survival beyond $24 \mathrm{~h}$ was recorded.

The rate of pup cannibalizations was $100 \%$ during Phase 1 and Phase 2; however, the longevity of infants decreased following the combining of populations. While pups often survived for 24 to $36 \mathrm{~h}$ in
Phase 1, during the period following the combining of populations, pups were rarely observed to survive beyong $24 \mathrm{~h}$. The observed occasions of infant cannibalization in Phase 2 were always coincident with postparturition estrus. During these periods, the lactating female would be incessantly pursued and mounted. As a result, the lactating female was chased out of the nest, the nest was repeatedly interrupted, pups were neither retrieved nor defended, and the opportunity for cannibalization was unrestricted. Only once was cannibalization by a lactating female observed; all other cannibalizations were executed by males and nonlactating females. Prior to the combining of populations, nest defense and pup retrieving were observed on a limited scale. After the 19th day of Phase 2, when litters were again present, neither nest defense nor pup retrieving was observed. During this later phase, the lactating female was mounted repeatedly, even before all her pups had been delivered, thereby physically prevented from any nest defense or maintenance.

Although the animals were observed for all types of behavior considered indicative of consummatory behavior in aggregate, this part of the behavioral sink phenomenon did not appear with respect to daily feeding. This may have been due to the normal feeding procedure, which consisted of spreading pellets over the cage floor rather than requiring the animals to feed from a community tray (see Calhoun, 1962). With respect to daily drinking, however, consummatory behavior in aggregate was observed. Although on an ad-lib water schedule, the subjects engaged in apparently nonaggressive competitive drinking whenever the water bottles were changed, even though the bottles removed occasionally contained water which had not been consumed. This behavior was first observed on December 8 and regularly thereafter. It was simple to produce the drinking aggregates. A bottle could be removed for a brief period and then reinserted; within seconds, as many as 10 animals competed for access to the tube, even though adjacent tubes with water were available. On the basis of these observations, Experiment 5 was designed to investigate feeding and drinking competition aspects of the behavioral sink.

\section{Discussion and Conclusions}

Aggression. The large increase in aggression between the final week of Phase 1 and first week of Phase 2 seems to be related to the bringing together of two populations with no mutually established social structure. Social density alone cannot be the cause of aggression in this study, since no difference in aggression was found in Phase 3 when the animals were separated (May 23-May 29) and then recombined (May 30-June 3). The most reasonable assumption is that the social order developed prior to May 23 continued after the recombination. 
Natality and Mortality. With respect to population reduction mechanisms, the sudden change in social conditions at the beginning of Phase 2 produced an immediate cessation of litter production as in the Bruce effect (1960), together with an increase in adult mortality. These effects with adults were limited to the first part of Phase 2, while the infant mortality rate continued at $100 \%$ throughout Phases 2 and 3 . The shorter latency of cannibalization of pups in Phase 2 as compared to Phase 1 could also be attributed to social disorganization resulting from combining the groups. Under the present experimental conditions, the cannibalization of pups prevented population expansion, but the relation to other types of population control is not clear; it could well be situation specific. The increase in adult mortality lasted only a short period, reinforcing the belief that social disorganization is a more important factor in producing stress-related behaviors than social density alone.

\section{EXPERIMENT 2: PARENTAL CARE}

Since the pups born during Experiment 1 did not survive, a sample of mothers was withdrawn to assess the possibility that the zero population growth had been due to a failure of parental care on the part of the mothers.

\footnotetext{
Method

Subjects and Apparatus. The subjects were 18 adult (190-dayold) females separated from the animals of Experiment 1 at the end of Phase 2 (January 8) and housed individually thereafter. The apparatus consisted of a standard metal rack of individual laboratory rat cages with water bottles and ad-lib pellet dispensers.

Procedures. The 18 females were maintained under ad-lib conditions, which included an abundant supply of torn paper towel strips. The subjects were checked daily for litters and a census was kept. The study began on January 8 and continued until April 1.
}

\section{Results and Discussion}

Thirteen of the 18 mothers gave birth to litters, and one cannibalized her litter upon birth. The average litter size at birth was nine pups, and an average of six pups per litter survived to the 21 st day. One of the mothers died when her litter was 2 weeks old, and four of her pups survived to the adult stage. A total of 70 pups survived to the juvenile stage, 37 females and 33 males.

The results of Experiment 2 indicate that the zero population growth of Experiment 1 was not due to a failure of parental care on the part of the mothers.

\section{EXPERIMENT 3: GROUP COMPOSITION, SPATIAL DENSITY - SOCIAL DENSITY, AND MALE-MALE AGGRESSION}

The purpose of this experiment was to assess the possibility that the $100 \%$ infant mortality of Experiment 1 might have been due to aggression between males and/or unknown limiting factors inherent in the restriction on available space.

\begin{abstract}
Method
Subjects and Apparatus. The subjects were 18 naive females from Simonsen Laboratories and one of the dominant males from the males of Experiment 1. The apparatus was a single cage, $3.11 \mathrm{~m}$ long, $1.26 \mathrm{~m}$ wide, and $0.46 \mathrm{~m}$ deep (.206 sq $\mathrm{m}$ per animal). Hardware cloth and lumber were used in constructing the cage, which, except for being one and a half times larger, was identical to the cage used in Phase 2 of Experiment 1.

Procedure. The subjects were maintained on ad-lib conditions with an abundant supply of nesting material of the same type used in Experiments 1 and 2. The composition of the group in this experiment precluded male-male aggression (1 male and 18 females). Thus both social and spatial density were lower than in Phase 1 or Phase 2 of Experiment 1 (social density of 171 vs. 351 or 1,431 , and $.037 \mathrm{vs} .206 \mathrm{sq} \mathrm{m}$ per animal). Also, the nature and quality of social relationships were importantly altered by the elimination of male-male interactions. The group was maintained for 120 days (February 8-May 21) and observed daily for pups.
\end{abstract}

\section{Results and Discussion}

Although litters of pups occurred regularly, all were cannibalized by the single male and the nonlactating females. Thus, elimination of male-male interactions and substantial decreases in social and spatial density were not sufficient to reduce cannibalism.

\section{EXPERIMENT 4: PROTECTED NEST CONDITIONS AND CRITICAL AGE}

The purpose of this experiment was to assess the effect of type of housing on pup mortality and to determine the critical age beyond which a pup can survive in a high-density environment. Since in the previous experiments of this series the mothers were not afforded a place in which to burrow, their nests were structurally unprotected. Because this may have been the effective variable in zero population growth, Experiment 4 was designed to examine pup natality and mortality under conditions in which the mothers were afforded a space in which the nest were structurally protected on four sides, and later on four sides and the top. This experiment was also designed to determine the age beyond which a pup could survive in a socially and spatially dense population. 


\section{Method}

Subjects. The subjects were 30 female and 30 male Long-Evans rats between 120 and 140 days of age. The females had been used previously in a learning study (McCain, Ward, \& Lobb, 1975). The males were survivors of the litters in Experiment 2.

Apparatus. The same 2-sq-m cage that was used in Phases 2 and 3 of Experiment 1 was employed as the housing apparatus. The only modification was the partitioning of one-half the cage into 20 individual housing units made of $1.3-\mathrm{cm}$ plywood and painted midgray. Each unit was $22.9 \mathrm{~cm}$ long and $15.2 \mathrm{~cm}$ wide. The bottom of the main cage was stapled to these partitions. The result of this modification to the main cage was that one side consisted of an open space of $1 \mathrm{sq} \mathrm{m}$, while the other half was divided into 20 adjacent cubicles.

Procedure. In Phase 1, the subjects were moved into the cage (June 7) and maintained under ad-lib conditions until July 17. Data collection consisted of observing for nest construction, nest location, and pups.

In addition to this colony group, six isolated females were maintained in typical laboratory racks and impregnated. The six mothers and litters were moved into the experimental cage, three with pups less than 10 days and one each at 10, 15, and 21 days of age. The litters were added on June 18, the survivors were removed on July 10.

In Phase 2, on July 17, a block of wood, $66 \times 10 \times 10 \mathrm{~cm}$, was placed across the top of the housing units, partially covering 7 of the 20 units. Phase 2 was terminated October 13.

\section{Results}

Prior to the beginning of Phase 2, 27 litters or remains of litters were observed; all pups were cannibalized within 2 days of birth. The reported number of litters is an estimate due to the possibility that litters were consumed overnight or between observation sessions. During this same period, the litters from the isolated mothers were added to the main colony. The three litters which had not yet developed body hair (less than 10 days old) did not survive; all of the pups with body hair when added survived until removed.

The survival rate of pups born in the main cage can only be estimated because of the possibility that some litters were born overnight and cannibalized before the following day's census was taken. All nests were shifted (by the mothers) overnight into units which were partially covered when the wood block was added. It was conservatively estimated that at least $50 \%$ of the pups born after the installation of the wood block covering (Phase 2) survived until the termination of the experiment 3 months later. During Phase 2, the population doubled; the final census consisted of 52 males, 62 females, and 12 pups. The net increase in adult population was 48 ; the population difference between adults in Phases 1 and 2 was significant $\left[\chi^{2}(1)=12.8, p<.001\right]$.

Figure 2 shows the results of the pup and adult census measures. The ordinate is expressed as the number of pups observed without hair, while the abscissa is expressed in days. The decrease in August is due to maturing of the pups.

\section{Discussion}

The decrease in the pup mortality rate is not surprising if consideration is given to the natural habitat of the wild rat which includes a protected burrow. Presumably, emigration prevents an excessive buildup of population in a single burrow in the wild. But the point of research such as the Calhoun studies is that emigration was blocked, and thus the confined population grows until some limiting factor arises. One question in this line of research concerns identification of the mechanisms by which the population limits itself when density exceeds the availability of burrow space. Regardless of the density level used in these studies, the cannibalization of pups in unprotected nests was a major factor in the control of population growth.

The defense reactions of a female rat with pups may require a fully formed and structurally protected burrow before defense reactions can function effectively. Under conditions of high social and/or spatial density, there is a shortage of available and defensible burrow space. Under these conditions, the mother would have to form a nest in a relatively open space or at least in a partially unprotected area. The female rat's defense reactions, which may be integral to a spatially protected nest in a burrow, apparently do not suffice for the necessary defense of a nest that is inadequately covered.

On each occasion of cannibalization of an adult, the skin and fur were not consumed. Furthermore, pups with hair were not cannibalized. These observations, along with the complete cannibalization of hairless pups, suggests that an active avoidance of hair may be involved. A replication of Experiment 4 with hairless adults (shaved) being introduced into the population would appear to be a timely and important study.

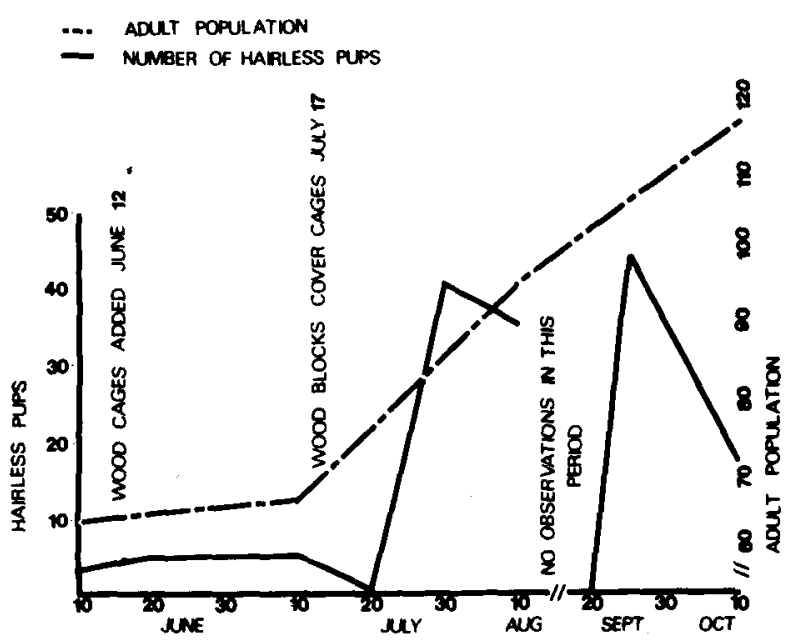

Figure 2. Procedures and results of Experiment 4 (see text). 


\section{EXPERIMENT 5: NONAGGRESSIVE COMPETITION}

During Phases 2 and 3 of Experiment 1, several behavioral observations appeared to fit the description of either a "feeding frenzy" or a food-related "behavioral sink" as reported by Calhoun. Although Phase 2 of Experiment 1 was not designed to produce the effect, drinking in aggregate was observed on numerous occasions. The behavior consisted of an orientation toward water nozzles and evidently was stimulated by the daily replacement of water bottles. An aggregate bout differs from ordinary drinking in four ways. First, the bouts, once initiated by a change in water bottles, persisted longer than normal bouts of solitary drinking. Second, the animals were distributed about the water bottle nozzles in a nonrandom array. That is, the number of animals drinking from each of the 14 available nozzles was neither uniformly nor randomly distributed; rather, the animals were clustered about some nozzles while other nozzles were vacant or had only one drinker. Third, the aggregates of competing animals were semicone shaped, with the apex oriented toward a water nozzle. Fourth, the animals taking part in these apexes would characteristically (1) squeal in a high-frequency, lowamplitude repetitive fashion that was similar to the vocalization of new-born nursing pups, and (2) touch the adjacent members of the apex in a rhythmic fashion unless the limbs being used did not contact another body, in which case the limbs would be fully extended. Squeals and touching were never observed when less than three animals were attempting to drink from one tube. The above characteristics were used as criteria for the identification of consummatory behavior in aggregate. The nonaggressive property of this behavior was experimentally measured.

\section{Method}

Subjects. The subjects were the same animals used in Experiment 1 .

Apparatus. The housing apparatus was described in Experiment 1 (Phases 2 and 3), and the system of 141 -liter water bottles was used as a fixed (nonmobile) water source. This system presented 14 water nozzles through one side of the housing cage with each nozzle $13 \mathrm{~cm}$ distant from the next. During the test for drinking in aggregate, these 14 nozzles were inserted through the cage wall, thus acting as a fixed water source. At other times, the nozzles were removed. The mobile water source consisted of two watersaturated cotton swabs which were placed in each of 14 jar lids that had been nailed to two boards, $100 \times 2.54 \times 1.25 \mathrm{~cm}$. These baby-food jar lids were fastened $13 \mathrm{~cm}$ apart, and during the tests for drinking in aggregate, two saturated swabs were placed in each lid. The mobile food source consisted of two pellets placed in each of the 14 mounted jar lids before inserting the two boards. The fixed food source consisted of wet mash which was packed into the jar lids before inserting the boards. The mash and pellets had equal dry weights.

Procedure. In addition to daily observations, possible consummatory behavior in aggregate during food and/or water competition was systematically measured during two 48-h periods, February 16 and 17, 1975, and June 2 and 3, 1975. No food or water was available other than that given in the test periods. Thus, the first measurements were taken in the early stage of Phase 2 of Experiment 1, while the second set of measurements were conducted during the terminal stage of Phase 3, Experiment 1. This procedure allowed for measurements of food and/or water competition under relatively high- and low-aggression conditions.

A condition of consummatory behavior in aggregate was scored when: (1) the bouts were long in duration, (2) the drinkers or eaters were distributed in a nonrandom array (i.e., at least one other lid or nozzle was vacant although it contained food or water), (3) the animals were in aggregates, and (4) they were squealing in a repetitive manner with rhythmic limb extension. The number of animals consuming from each nozzle or lid was recorded every $2 \mathrm{~min}$.

Each day, data were collected in four 16-min tests, with a different condition (food or water; fixed or mobile) in effect during each 16-min test period. Twelve hours later, all four conditions were given again, but in a different order. The four separate conditions were (1) fixed water (the nozzles), (2) mobile water (two wet cotton swabs in each of the jar lids), (3) fixed food (each of the jar lids packed with wet mash), and (4) mobile food (each of the jar lids containing two lab pellets of equal weight to the dry weight of the mash). The cotton swabs and the pellets were not replaced after consumption.

The consummatory behavior in-aggregate tests were conducted $0,6,12,24,36$, and $48 \mathrm{~h}$ after all other sources of food and water had been removed. Thus, a successive 12-h deprivation schedule (except for the one 6-h point) with 16 min restricted ad-lib feeding was in effect. In these tests, a condition was presented and the animals engaged in consummatory behavior in aggregate at each nozzle or jar lid during each successive 2 -min period were were counted. The frequency of aggressive behaviors was also recorded during each of the eight successive 2 -min periods during each test. Data collection of June 2 and 3 was carried out with the February 16 and 17 procedures, except that the 6-h point was omitted inadvertently. A different experimenter was used in the data collection for February 16 and 17 and June 2 and 3.

\section{Results}

At least two indications support the hypothesis that competitive drinking in aggregate is related to the fixed water source condition. First, the animals met the criteria for the consummatory behavior in aggregate mentioned earlier. Second, as can be seen in Table 1, the animals form groups of clusters about

Table

Number of Animals at a Fixed Goal Source During the First $2 \mathrm{~min}$ of Data Collection for Each Deprivation Condition

\begin{tabular}{|c|c|c|c|c|c|c|c|c|c|c|c|c|c|c|}
\hline \multirow[b]{2}{*}{ Hours } & \multicolumn{14}{|c|}{ Position of Food Cup or Nozzle } \\
\hline & 1 & 2 & 3 & 4 & 5 & 6 & 7 & 8 & 9 & 10 & 11 & 12 & 13 & 14 \\
\hline \multicolumn{15}{|c|}{ Fixed Water } \\
\hline 0 & 1 & 1 & 1 & 2 & 2 & 3 & 4 & 0 & 4 & 1 & 1 & 1 & 3 & 1 \\
\hline 12 & 3 & 2 & 4 & 4 & 3 & 2 & 4 & 0 & 5 & 3 & 0 & 4 & 3 & 0 \\
\hline 24 & 4 & 2 & 4 & 4 & 5 & 7 & 5 & 6 & 0 & 5 & 2 & 2 & 1 & 1 \\
\hline 36 & 3 & 2 & 5 & 2 & 5 & 3 & 4 & 4 & 4 & 5 & 4 & 3 & 4 & 2 \\
\hline 48 & 7 & 4 & 5 & 4 & 6 & 7 & 7 & 3 & 3 & 2 & 0 & 0 & 0 & 0 \\
\hline \multicolumn{15}{|c|}{ Fixed Food } \\
\hline 0 & 2 & 2 & 0 & 1 & 3 & 2 & 3 & 3 & 3 & 2 & 0 & 2 & 0 & 0 \\
\hline 12 & 0 & 1 & 1 & 2 & 1 & 2 & 2 & 6 & 6 & 8 & 5 & 6 & 3 & 4 \\
\hline 24 & 3 & 3 & 3 & 2 & 4 & 2 & 3 & 4 & 5 & 4 & 2 & 4 & 6 & 4 \\
\hline 36 & 1 & 1 & 2 & 3 & 2 & 1 & 2 & 6 & 3 & 4 & 8 & 6 & 5 & 3 \\
\hline 48 & 2 & 2 & 1 & 1 & 1 & 2 & 3 & 6 & 9 & 9 & 5 & 2 & 5 & 6 \\
\hline
\end{tabular}

Note-Clusters of animals in competition are shown in italics. 
some food or water sources while other sources are vacant or consumed from without competition. All drinking, whether in aggregate or not, declined rapidly after about $8 \mathrm{~min}$ of each test.

Although the consummatory behavior in aggregate was clearly present during the first 2 min of each fixed food test, the behavior declined rapidly as the food source was consumed. Since, in this experimental design, no provisions were made for renewing the food source, the usable data are limited to the first $2 \mathrm{~min}$, and thus these results may be considered sufficient only to suggest the existence of competitive feeding in aggregate. However, the goodness-of-fit tests tor differences in frequency of eaters in aggregate were significant (see Table 1). If these procedures are considered to be an acceptable replication of the Calhoun feeding procedure, then the Calhoun report on a feeding related behavioral sink is supported.

Figure 3 shows the proportion of animals' consummatory behavior in aggregate at each of the deprivation points. The frequencies of observed aggressive behavior during each of these test periods are shown in Figure 4. Excluding the zero deprivation point, which does not distinguish between measures, the frequency of aggression is consistently greater with mobile food than with any other condition. Various bases of calculation on the data in Figure 4 all gave significant differences $(p<.05)$ by application of a probability test. Thus, consummatory behavior in aggregate appears to be a form of nonaggressive competition.

\section{Discussion}

The first and most obvious result of these manipulations is that a fixed food or water source is more likely to produce nonaggressive competition and consummatory behavior in aggregate than is a mobile source. Although the assumption that a wet cotton swab constitutes a mobile water source may be

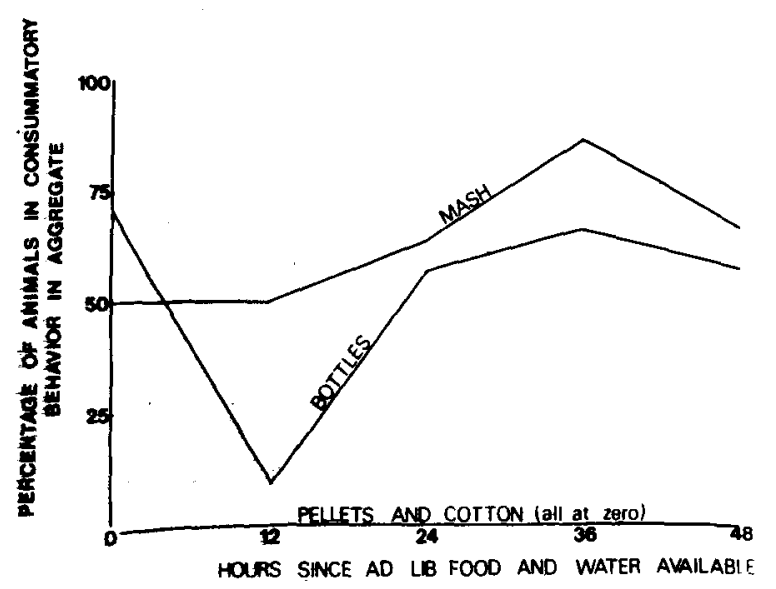

Figure 3. Conoumnatory behavior in agregate of Experiment 5.

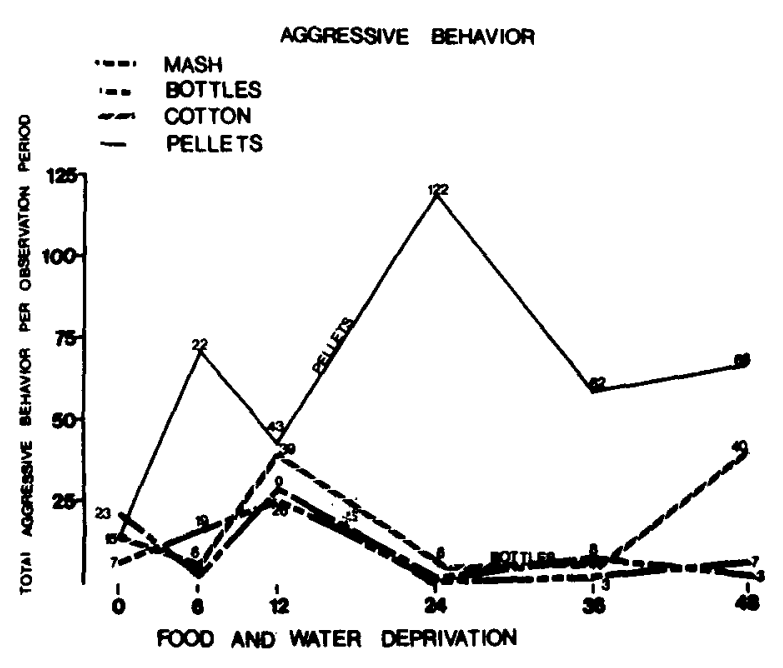

Figure 4. Aggressive behavior with mobile and nonmobile goal sources (Experiment 5).

questionable, the repeated observation of a nonaggressive competition in aggregate with fixed food and water is clear and convincing. Since fixed food produced dense aggregations with low aggression, and since mobile food produced very minimal aggregations with high aggression, the competitive behavior in aggregate can be characterized as nonaggressive. The fixed water condition also appears to result in nonaggressive competition. Thus the behavior being reported here may be described as a competitive but nonaggressive social orientation toward and consumption from a fixed goal source.

Although a fixed goal source may be a necessary condition, it may not be sufficient for a demonstration of nonaggressive competition. The possibility of experience on mild deprivation during the period prior to testing cannot be excluded, and indeed deprivation in a group of animals consuming from a fixed source may be necessary for most demonstrations of nonaggressive competition. Furthermore, because the form or pattern of the behavior is constant, it may be an example of a genetically predisposed behavior which could be related to nursing. The attachment of this fixed behavioral form to water nozzles and food cups may require deprivation and the repeated experience of goal consummation from limited resources during which the fixed behavior is conditioned to the nozzles and cups. This hypothesis might be tested by rearing single and multiple pup litters and comparing adult behavior during consumption from limited resources under deprivational conditions.

An alternative explanation of these data may be constructed from the standpoint of animal orientation. In the fixed-source condition, the orientation of the animals is primarily directed toward the goal object, while in the mobile-source condition, the orientation is shifted toward the animal carrying the goal object. 
The shift in orientation may predispose the form of the ensuing behavior, i.e., aggressive or nonaggressive competition.

Perhaps the most important result of these manipulations, regardless of explanation, is that some of the behaviors (in aggregate), referred to as a part of the behavioral sink phenomenon or alternately the feeding frenzy, can now be reproduced systematically in the laboratory.

\section{GENERAL DISCUSSION}

These experiments support four principal conclusions. First, either social density and/or social disorganization, and not spatial density per se, was an effective agent related to stress of population density in this study. However, an investigation which systematically varies spatial density under constant social density is needed. Second, cannibalization of hairless pups in indefensible nests is a major population control mechanism under several conditions. Third, nonaggressive competition does exist and can be reproduced by manipulations in the laboratory. And fourth, competition for limited resources does not necessarily include aggressive behavior.

Several hypotheses are suggested by these results. First, the pattern of behavior seen in the consummatory behavior in aggregate may first appear in infancy during nursing and subsequently be conditioned to nozzles and food cups. This suggestion is, in part, based on the apparent similarity of form between pawing/squealing patterns in infant nursing and adult consummatory behavior in aggregate. Second, population stabilization may proceed very rapidly when nests are not defensible. Here the exact relation of social density is not clear. Third, aggressionbased territorial exclusion may be an insufficient population reduction mechanism. If territorial exclusion were effective, then it should be most apparent during or in association with consuming from limited fixed sources where significantly less aggression was observed. Fourth, hair may act as a barrier to cannibalism.

A replication of the present procedures under controlled field conditions with an indigenous group of rodents with laboratory replication is clearly needed.

\section{REFERENCE NOTE}

1. Fidell, S. L., Lopez, A., \& Waddell, C. Physiological and behavioral reactions of rats to stress of overcrowding. Unpublished paper, San Fernando Valley State College, 1972.

\section{REFERENCES}

Altman, I. The environment and social behavior: Privacy, personal space, territory and crowding. Monterey, Calif: Brooks/Cole, 1975.

Barnett, S. A., \& Evans, C. S. Questions on the social dynamics of rodents. In P. D. Ellis (Ed.), Social organization of animal communities. Zoological Society of London. Symposia, 26-27 November, 1963.

BRUCE, H. M. A block of pregnancy in the mouse caused by proximity of strange males. Journal of Reproductive Fertility, $1960,1,96-103$

Calhoun, J. B. Population density and social pathology. Scientific American, 1962, 306, 139-148.

Carpenter, C. R. A field study in Siam of the behavior and social relations of the gibbon [Hylobates lar). In C. H. Southwick (Ed.), Primate social behavior. Princeton, N.J: Van Nostrand, 1940 and 1963.

Cox, V. C., Paulus, P. B., McCain, G., \& Schimade, J. K. Field research on the effects of crowding in prisons and on offshore drilling platforms. Human Ecology, in press. (To appear in J. R. Aiello (Ed.), Residential crowding and design. New York: Plenum Press.)

Eibl-Eibesfeldt, I. The fighting behavior of animals. Scientific American, 1961, 205, 112.122.

EsSER, A. H. (Ed.) Behavior and environment: The use of space by animals and man. New York: Plenum Press, 1971.

MCCAIN, G., WARD, R., \& LoBB, M. Reward magnitude and a comment. Bulletin of the Psychonomic Society, 1975, 7, 90-92.

Paulus, P., Cox, V., McCain, G., \& Chandler, J. Some effects of crowding in a prison environment. Journal of Applied Social Psychology, 1975, 5, 86-91.

STokols, D. A social-psychological model of human crowding. Journal of American Institute of Planners, 1972, 38, 72-84.

Wohlwll, J. F., \& CARson, D. H. (Eds.) Environment and the social sciences: Perspectives and applications. Washington, D.C: American Psychological Association, 1972.

\section{NOTE}

1. Pilot work was begun on August 25, 1974, and continued until the actual study began on October 23, 1974, and terminated on October 10,1975 . In this paper, some of the manipulations (e.g., Experiment 5 and Experiment 2) are presented as if they were separate experiments in spite of the fact that they were conducted within the context of Experiment 1. Some of the manipulations (e.g., consummatory behavior in aggregate and the second separation and recombination) were not anticipated when the study was designed. These were performed because the potential information seemed useful.

(Received for publication February 16, 1977; revision accepted October 20, 1977.) 